\title{
Peripheral Neurological Disturbances, Autonomic Dysfunction, and Antineuronal Antibodies in Adult Celiac Disease Before and After a Gluten-Free Diet
}

\author{
Antonio Tursi - Gian Marco Giorgetti • Cesare Iani · \\ Flavio Arciprete • Giovanni Brandimarte • \\ Ambrogio Capria · Luigi Fontana
}

Received: 15 July 2005 / Accepted: 13 September 2005 / Published online: 12 September 2006

(C) Springer Science+Business Media, Inc. 2006

\begin{abstract}
Thirty-two consecutive adult celiac disease (CD) patients (pts), complaining of peripheral neuropathy (12 pts), autonomic dysfunction (17 pts), or both (3 pts), were evaluated to assess the presence of neurological damage (by clinical neurological evaluation and electrophysiological study) and antineuronal antibodies and to assess the effect of a gluten-free diet (GFD) on the course of the neurological symptoms and on antineuronal antibodies. At entry, 12 of $32(38 \%)$ pts showed signs and symptoms of neurological damage: 7 of $12(58 \%)$, peripheral neurological damage; 3 of $12(25 \%)$, autonomic dysfunction; and 2 (17\%), both peripheral neurological damage and autonomic dysfunction. The
\end{abstract}

\section{A. Tursi}

Digestive Endoscopy Unit, Lorenzo Bonomo Hospital,

Andria, (BA)

G. M. Giorgetti

Department of Internal Medicine, Clinical Nutrition Unit,

S. Eugenio Hospital,

Rome, Italy

C. Iani - F. Arciprete

Division of Neurology, S. Eugenio Hospital,

Rome, Italy

G. Brandimarte

Department of Internal Medicine, Division of Gastroenterology, Cristo Re Hospital,

Rome, Italy

\section{A. Capria $\cdot$ L. Fontana}

Department of Internal Medicine and Immunology,

Tor Vergata University,

Rome, Italy

\section{A. Tursi $(\square)$}

Galleria Pisani 4, 70031 Andria (BA),

Italy

e-mail: antotursi@tiscali.it. overall TNS score was 105 at entry. Anti-GM1 antibodies were present in 5 of 12 (42\%) pts: 3 showed peripheral neurological damage and 2 showed both peripheral neurological damage and autonomic dysfunction. One year after the GFD was started, histological lesions were still present in only 10 of $12(83 \%)$ pts. TNS score was 99, 98, 98, and 101 at the 3rd, 6th, 9th, and 12th month after the GFD was started, so it did not improve throughout the follow-up. None of the pts showed disappearance of antineuronal antibodies throughout the follow-up. We conclude that adult CD patients may show neurological damage and presence of antineuronal antibodies. Unfortunately, these findings do not disappear with a GFD.

Keywords: Antineuronal antibodies - Autonomic dysfunction - Celiac disease · Gluten-free diet · Peripheral neuropathy

Celiac disease (CD) is a chronic inflammatory disease of the gut occurring in genetically susceptible individuals after ingestion of gluten. It is characterized by a flattened mucosa, villous atrophy, and crypt hyperplasia in the small intestine and by classic malabsorption syndrome (diarrhea, steatorrhea, weight loss) or by minor, apparently unrelated symptoms such as iron-deficiency anemia, osteopenic bone disease, amenorrhea, and infertility [1]. The absence of gluten in the diet generally leads to a return to normality of the morphological changes [2].

Untreated CD may show various neurological manifestations such as cerebellar ataxia, epilepsy, and brain atrophy [3-7]. Moreover, associations among $\mathrm{CD}$, peripheral neuropathy, and neuromuscular disorders have been described [8-10]. These were assumed to be due to malabsorption, although nutritional deficiencies were rarely demonstrated, and no improvement of the neurological disease was 
observed following vitamin therapy $[11,12]$. As CD is known to be linked to autoimmunity [13, 14], it is hypothesized that antineuronal antibodies may provoke these lesions.

The aim of our study was thus to evaluate neurologically a cohort of untreated adult CD patients, to evaluate the presence of antineuronal antibodies, and to evaluate the effect of a gluten-free diet (GFD) on the clinical course of the neurological disease.

\section{Methods}

We studied 32 consecutive adult CD patients in whom CD was diagnosed for the first time ( 7 males and 25 females; mean age, 32.7 years; range, 17-73 years). All of them complained of suspected peripheral neuropathy (paresthesia of arms and/or legs, reduction of muscular strength; 12 patients), or autonomic dysfunction (vertigo, weakness, presyncope, recurrent syncope, postural nausea; 17 patients), or both (3 patients) $[15,16]$. None of the patients suffered from other autoimmune disease or from diseases affected by neurological complication (such as diabetes).

Both the original [17] and the revised [2] criteria for the diagnosis of $\mathrm{CD}$ were used in this study.

Endoscopic procedures Esophagogastroduodenoscopy (Fujinon EG300 videogastroscope; Fujinon, Omiya, Japan) was performed in all patients, who also underwent small bowel biopsy. At least six small bowel biopsies were obtained from the second part of the duodenum and evaluated by hematoxylin/eosin staining. Obviously, bioptic samples to detect villous atrophy were obtained not only in patients with an endoscopic aspect suspicious for $\mathrm{CD}$ but also in patients with a normal endoscopic aspect and suspected to be affected by CD. CD was defined as permanent gluten-sensitive enteropathy, primarily expressed by the presence of characteristic small intestinal lesions [18].

Histological classification Histopathology was expressed according to the Marsh classification modified by Oberhuber [19]: "infiltrative" lesions with $>30$ lymphocytes/100 epithelial cells are defined as Marsh type I, "infiltrative/ hyperplastic" lesions as Marsh II, and "partial (sub)total villous atrophy (VA) as type III. We subdivided the Marsh III type into partial VA (Marsh IIIa), subtotal VA (Marsh IIIb), and total VA (Marsh IIIc).

Other possible causes of villous atrophy or duodenal damage, such as Giardia lamblia infestation, tropical sprue, collagenous sprue, and food protein hypersensitivity (cow's milks, eggs, fish, rice, chicken), were excluded, as well as other causes of inflammatory infiltration of the duodenum, such as peptic duodenitis [19].
Other noninvasive diagnostic methods In all patients we also performed $\mathrm{H}_{2}$ sorbitol breath test and anti-tTG antibody and endomysial antibody (EMA) evaluation at the time of diagnosis (before the GFD was started) and 6 and 12 months after the GFD was started.

IgA antigliadin antibodies (AGAs) were measured in all patients by enzyme-linked immunosorbent assay (ELISA; Alfa-gliatest kit; Eurospital, Trieste, Italy); the lower limit of positivity of the $\operatorname{IgA}$ class was $0.2 \mathrm{EU} / \mathrm{ml}$. IgA anti-tissue transglutaminase antibodies were determined by ELISA using human recombinant tTG (Eu-tTG kit; Eurospital); the lower limit of positivity of these antibodies was $7 \mathrm{AU} / \mathrm{ml}$. IgA EMAs were screened by the indirect immunofluorescent method on monkey esophagus (Antiendomysium kit; Eurospital).

Also, a sorbitol $\mathrm{H}_{2}$ breath test was performed in all patients to assess malabsorption. The patients were studied after an overnight fast, having been instructed to consume a meal of rice and meat; they were requested not to smoke on the morning of the test. End expiratory samples were collected before the patients drank the test solution ( $5 \mathrm{~g}$ of sorbitol in $150 \mathrm{ml}$ of tap water) and every $30 \mathrm{~min}$. for $4 \mathrm{hr}$. Hydrogen concentrations in all collected samples were measured with a breath hydrogen analyzer (EC60 Gastrolyzer Breath Hydrogen Monitor; Bedfont Scientific Ltd., Upchurch, Kent, UK). An increase in $\mathrm{H}_{2}$ concentration of at least $20 \mathrm{ppm}$ over the fasting baseline was considered positive for sorbitol malabsorption. The cutoff for calculating the validity of the test was shifted every $30 \mathrm{~min}$, and a response operating characteristic (ROC) curve was plotted on the basis of the results obtained.

Neurological study All patients were investigated by clinical neurological evaluation and by electrophysiological study. For this study, (i) clinical neurological evaluation and (ii) electrodiagnostic evaluation were performed before the GFD and every 3 months after the GFD was started.

Neurological evaluation consisted of the following.

1. Symptoms referred by patients. These were classified as suspicious for peripheral nervous system dysfunction (paresthesia of arms and/or legs, reduction of muscular strength) or for autonomic dysfunction (vertigo, weakness, presyncope, recurrent syncope, postural nausea).

2. Signs and symptoms obtained from neurological examination (tactile- and thermal-painful sensitivity, evaluation of muscular strength).

3. Evaluation of specific neuromyographic parameters; nerve conduction study was carried out in motor (peroneal nerve) and sensory (sural nerve) nerves.

All results were evaluated according to Total Neuropathy Score (TNS) [20] and according to Medical Research Council criteria. 
Electrodiagnostic evaluation was performed to assess motor or sensitive neurological disturbances in our celiac population. Thus, we performed the following evaluations.

1. Evaluation of four motor nerves (left median, ulnar, peroneal, tibial nerves) and four sensitive nerves (left median and ulnar nerves [orthodromic study], radial and sural nerves [antidromic study]).

2. Electroneuromyography (ENMG) was carried out on two proximal muscles (deltoid from upper limbs and vastum lateralis from lower limbs). As a rule, the ENMG was performed to the left extremities, while the right extremities were examined in patients with predominantly right-sided symptoms. The ENMG was considered abnormal if fibrillation, positive sharp waves, or both were present at more than one location within the muscle.

3. Evaluation of the cutaneous-sympathetic response from the four limbs.

4. Assessment of extrinsic autonomic neuropathy by the standardized measurement of cardiovascular reflexes (heart rate variation [R-R interval] and systolic and diastolic blood pressure to a variety of stimuli: lying-tostanding, Valsalva maneuver, deep breathing, sustained handgrip, tilt test).

All the latter evaluations were performed using a Keypoint 4 electromyograph (Medtronic Functional Diagnostics). Finally, all patients underwent cerebral computerized tomography $(\mathrm{CT})$ to exclude cerebral causes of their symptoms.

Antineuronal antibody assessment The presence of antineuronal antibodies was detected in all patients studied by ELISA according to the criteria of the Italian Association of Neuro-Immunology (AINI) [21]. Microwells of ELISA plates were coated with $100 \mu \mathrm{l}$ of methanol containing $0.5 \mu \mathrm{l}$ of gangliosides (GM1 and GQ1b; Sigma Chemical Co., St. Louis, MO, USA) and antigens, and the methanol was allowed to evaporate. After saturation with $1 \%$ bovine serum albumin (BSA) in phosphate-buffered saline, increasing dilutions of patients' sera were added in triplicate and incubated at $4{ }^{\circ} \mathrm{C}$, followed by peroxidase-conjugated goat anti-human $\operatorname{IgM}, \operatorname{IgG}$, and $\operatorname{IgA}(0.8 \mathrm{mg} / \mathrm{ml})$ (Jackson Immunoreseach Laboratories, West Grove, PA, USA) diluted at 1:1000 for $2 \mathrm{~h} r$. Sera added to microwells coated with BSA only served as the control for each dilution. After washing, $100 \mu \mathrm{l}$ of developing solution containing 0.05 $\mathrm{M} \mathrm{Na} \mathrm{HPO}_{4}, 0.024 \mathrm{M}$ sodium citrate $\mathrm{pH} 5,0.08 \%$ ophenylenediamine, and $0.008 \% \mathrm{H}_{2} \mathrm{O}_{2}$ was added. Reaction products were measured spectrophotometrically at $450 \mathrm{~nm}$ in a Biotek reader. The titer for each specimen was taken as the highest dilution at which the optical density reading was 0.1 units higher than in the corresponding BSA-coated wells.

\section{Results}

Small bowel parameters before and after GFD

Histology

Histology improved significantly throughout the follow-up. Before starting the GFD, histological lesions were present in all patients (Marsh II lesion in one patient, Marsh IIIa in two, Marsh IIIb in five, and Marsh IIIc in four patients). One year after the GFD was started, histological lesions were still present in 10 of $12(\mathbf{8 3} \%)$ patients, but were less severe (Marsh 0 was present in 1 patient, Marsh I in 3, Marsh II in 4, Marsh IIIa in 3, Marsh IIIb and IIIc in 0 patients). These data are in accordance with our recent experience with long-term histological follow-up of $C D$ on GFD [22].

\section{Serology}

AGAs were present in 9 of 12 (75\%) patients, and EMA $s$ and anti-tTG in 10 of $12(\mathbf{8 3} \%)$ patients, before starting the GFD. One year after the GFD was started, AGAs were present in 2 of $12(\mathbf{1 7} \%)$ patients, and EMAs and anti-tTG were present in 1 of 12 patients $(\mathbf{8} \%)$.

\section{Sorbitol $\mathrm{H}_{2}$ breath test}

The test was positive in 11 of $12(92 \%)$ patients at entry, whereas it was positive in 6 of $10(60 \%)$ patients with persisting histological lesions.

Neurological assessment before and after the GFD

None of the 32 studied patients showed cerebral alterations on CT scan.

Regarding assessment of neurological damage, only 12 of $32(38 \%)$ patients showed signs and symptoms of neurological damage ( 2 males and 10 females; mean age, 41.25 years; range, 32-58 years): 7 of 12 (58\%) patients showed peripheral neurological damage, 3 of $12(25 \%)$ patients showed autonomic dysfunction, and 2 patients (17\%) showed both peripheral neurological damage and autonomic dysfunction. The overall TNS score was 105 at entry and 99, 98, 98, and 101 at 3, 6, 9, and 12 months, respectively, after the GFD was started; so it did not improve throughout the follow-up. Moreover, no difference in TNS score was found between patients affected by peripheral neuropathy and those with autonomic neuropathy before and after the GFD.

Evaluation of antineuronal antibodies reveealed that antiGM1 antibodies were present in 5 of $12(42 \%)$ patients: 3 patients showed peripheral neurological damage and 2 patients showed both peripheral neurological damage and autonomic dysfunction. So, none of the patients affected by autonomic 
Table 1. Characteristics of CD patients found to be affected by neurological damage*

\begin{tabular}{llllll}
\hline Sex & Age & Peripheral neurological damage & $\begin{array}{l}\text { Autonomic } \\
\text { dysfunction }\end{array}$ & Anti-GM1 & Anti-GQ1b \\
\hline F & 36 & Sensitive mononeuropathy of the radial nerve & No & Absent & Absent \\
F & 33 & Sensitive mononeuropathy of the radial nerve & No & Absent & Absent \\
F & 58 & Mononeuropathy of the tibial nerve & No & Present & Absent \\
F & 37 & Mononeuropathy of the tibial nerve plus myogenic damage of & No & Absent & Absent \\
& & the proximal muscles of lower limbs & & & \\
F & 33 & Axonal mononeuropathy of the tibial nerve & No & Absent & Absent \\
F & 37 & Sensitive axonal polineuropathy of the lower limbs & No & Present & Absent \\
M & 47 & Axonal-sensitive polineuropathy & Yes & Present & Absent \\
F & 32 & Axonal-sensitive polineuropathy & No & Present & Absent \\
M & 37 & Proximal, axonal polineuropathy & Yes & Present & Absent \\
F & 40 & None & Yes & Absent & Absent \\
F & 58 & None & Yes & Absent & Absent \\
F & 47 & None & Yes & Absent & Absent \\
\hline
\end{tabular}

*See text for further explanation.

dysfunction alone showed the presence of antineuronal antibodies (see also Table 1).

Finally, none of the patients showed the disappearance of antineuronal antibodies throughout the follow-up on GFD.

\section{Discussion}

Several studies have evaluated the possible neurological complications of $\mathrm{CD}$, but most of them have emphasized central nervous system involvement. On the contrary, peripheral nervous system (in particular, small fiber neuropathy [SFN]) involvement seems to play a more important role in the pathogenesis of neurological complications of CD. SFN is a neuropathy selectively involving small-diameter myelinated and unmyelinated nerve fibers. Interest in this disorder has increased considerably during the past few years. It is often idiopathic and typically presents with peripheral pain and/or symptoms of autonomic dysfunction. Diagnosis is made on the basis of the clinical features, normal nerve conduction studies (NCSs), and abnormal specialized tests of small nerve fibers. Among others, these tests include assessment of epidermal nerve fiber density, temperature sensation tests for sensory fibers, and sudomotor and cardiovagal testing (QSART) for autonomic fibers. Unless an underlying disease is identified, treatment is usually symptomatic and directed toward alleviation of neuropathic pain.

Association of neuropathy or myopathy with $\mathrm{CD}$ has been described infrequently. Holmes et al. analyzed neurological manifestations in a community-based CD population, and myopathy was diagnosed in $1.3 \%$ of cases (all of them resulting from osteomalacia) [23]. Another case report described a middle-aged man with a 15 -year history of $\mathrm{CD}$ who developed a large-fiber axonopathy affecting mainly sensory fibers [9]; in addition, inflammatory cell infiltrate was dis- covered in the nerve roots of the spinal cord in a CD patient with polyneuropathy [11]. Two large studies investigated peripheral neuropathy in $\mathrm{CD}[10,24]$. A more recent, and the largest, study, performed by Luostarinen et al., showed that polyneuropathy associated with $\mathrm{CD}$ is of the axonal type and affects both motor and sensory fibers; these authors also demonstrated that CD patients showed higher heat pain and touch thresholds than controls [24]. The very important finding of this study is that it was performed in patients who adopted a GFD and had CD in good remission, yet the patients still had an increased risk of clinical or subclinical neuropathy despite good adherence to the GFD [24]. These data reinforce the previous report of Volta et al., in which peripheral nervous disorders persisted in a 46-year-old female but improved significantly in a 38 -year-old female despite a GFD [25].

Similar results have been described for the association between $\mathrm{CD}$ and antineuronal antibodies. Their prevalence ranges from 22.22 to $61 \%$ in adults $[12,24]$, whereas in children the prevalence is about $5 \%$ [26]. The interesting finding of these reports is that in most cases these antibodies did not disappear after adoption of a GFD (except in a child reported by Briani et al. [26]).

The pathophysiology of these neurological dysfunctions in $\mathrm{CD}$ is unclear. One hypothesis suggests an overt or even subclinical malabsorption causing deficiencies of nutrients known to exert neurotrophic and neuroprotective effects (folic acid and vitamin $\mathrm{B}_{12}$ ) [8]. Selective vitamin $\mathrm{E}$ deficiency has been suggested as a potential cause of neurological complications in CD [27]; in addition, neurological involvement in CD may arise as a consequence of an autoimmune mechanism mediated by antigliadin antibodies $[4,7]$. Also, the significance of autoreactivity to gangliosides in CD is unclear, but it is probable that it may play a direct role in peripheral neuropathy [12]. 
In light of these literature reports, our study comprises several interesting findings. This is the first study investigating systematically a consecutive series of adult CD patients complaining of neurological peripheral or autonomic damage. A very high percentage of them showed clinical and electrophysiological signs of peripheral nervous damage, both neuromuscular and sensory (12 of 32 [37.5\%] evaluated patients). Moreover, some of them showed both peripheral neuropathy and autonomic damage ( 2 of 32 [6.25\%] evaluated patients). This is a very important finding, since this is the first report describing this wide neurological damage in CD patients.

Another interesting finding is that most of the patients affected by neurological disorders showed the presence of antibodies against gangliosides $(41.66 \%)$, in line with recent literature data on the prevalence of antiganglioside antibodies in adult CD patients [12, 24, 25].

But the very important finding of this study is that both neurological/autonomic damage and antibodies persisted despite strict adherence to the GFD (none of the patients reported violations of the GFD) and despite a good histological response to the GFD. This is the key point of this study, since we showed for the first time the ineffectiveness of a GFD in obtaining remission of neurological damage in well-treated $\mathrm{CD}$. Literature data showed that some cases of neurological damage in CD may regress after a GFD. Volta et al. described seven cases of neurological damage in $\mathrm{CD}$ with significant or even complete resolution of neurological symptoms after a GFD [25]. On the other hand, in the same report the authors described four cases of long-standing neurological disorders not responding to a strict GFD [26]. The latter finding is confirmed by the recent report of Luostarinen et al., who showed neuromuscular and sensory disturbances in patients with good compliance to a GFD [24].

At the same time, our data on antineuronal antibodies are quite different from the literature reports. Volta et al. described the disappearance of antibodies within 1 year in most patients, as well as the regression of these antibodies in pediatric CD patients [26]. On the contrary, in none of our celiac patients did we note the disappearance of antineuronal antibodies, and they seem to correlate with persistence of the neurological picture.

Why did neurological and/or autonomic dysfunction persist in our CD population after the GFD has started? Whatever the pathophysiology of neurological dysfunction and the significance of autoreactivity to gangliosides in CD may be, the first hypothesis is that the follow-up period of the study (12 months) may be sufficient for mucosal recovery but not for gluten-associated pathological conditions. However, it is probably that persistence of antibodies, as well as persistence of neurological symptoms, may be related to duration of gluten exposure. We can hypothesize a first step of neurological disease in $\mathrm{CD}$ when it is still gluten-sensitive. In this step a GFD may still effect both regression of neurological symptoms and disappearance of antineuronal antibodies. The following step may be considered gluten insensitive; both neurological symptoms and antineuronal antibodies persist despite a GFD, probably due to discharge of autoimmunity from gluten. The duration of gluten ingestion may thus be the key to explain this GFD insensitivity. Looking at the results of this study, we can see that the mean age of CD patients affected by peripheral neurological damage and/or autonomic dysfunction is quite high (40.73 years; range, $32-58$ years). It is thus hypothesized that this high mean age (and then the long-standing gluten exposure) may explain why neurological signs and symptoms persisted throughout the follow-up. This also may explain why we also diagnosed autonomic dysfunction in 4 of $32(33.33 \%)$ patients, whereas in a recent pilot study we did not find any sign of autonomic dysfunction in CD [28]. The duration of gluten exposure may thus be the key factor to explain our results, and this seems to be confirmed by other data, as the risk of developing autoimmune disorders is higher after long-standing gluten exposure [29, 30].

We conclude that some adult CD patients complaining of neurological symptoms show neurological damage as well as antineuronal antibodies, and that these findings do not disappear upon adoption of a GFD. Since the duration of gluten exposure is a possible explanation, our advice is to try to diagnose $\mathrm{CD}$ as early as possible, since delays in the diagnosis may cause severe and irreversible damage.

\section{References}

1. Martucci S, Biagi F, Di Sabatino A, Corazza GR (2002) Celiac disease. Dig Liver Dis 34(Suppl 2):S150-S153

2. Working Group of the United European Gastroenterology week in Amsterdam, 2001 (2001) When is a celiac a celiac? Eur J Gastroenterol Hepatol 13:1123-1128

3. Cronin CC, Jackson LM, Feighery C, et al. (1998) Celiac disease and epilepsy. Q J Med 91:303-308

4. Hadjjvassiliou M, Gibson A, Davies-Jones GAB, Lobo AJ, Stephenson TJ, Milford-Ward A (1996) Does cryptic gluten sensitivity play a part in neurological illness? Lancet 347:369371

5. Hadjjvassiliou M, Grunewald RA, Chattopadhyay AK, et al. (1998) Clinical, radiological, neurophysiological and neuropathological characteristics of gluten ataxia. Lancet 352:1582-1585

6. Pellecchia MT, Scala R, Filla A, De Michele G, Ciacci C, Barone $P$ (1999) Idiopathic cerebellar ataxia associated with celiac disease: lack of distintive neurological features. J Neurol Neurosurg Psychiatry 66:32-5

7. Collin P, Pirttilä T, Nurmikko T, Somer H, Erila T, Keyrilainen O (1991) Celiac disease, brain atrophy, and dementia. Neurology 41:372-375

8. Cooke WT, Smith T (1966) Neurological disorders associated with adult celiac disease. Brain 89:683-722

9. Kaplan JG, Pack D, Horoupian D, DeSouza T, Brin M, Schaumburg H (1988) Distal axonopathy associated with chronic gluten enteropathy: a treatable disorder. Neurology 38:642-645 
10. Hadjivassiliou M, Chattopadhyay AK, David-Jones GA; Gibson A, Grunenwald RA, Lobo AJ (1997) Neuromuscular disorder as a presenting feature of celiac disease. J Neurol Neurosurg Psychiatry 63:770-775

11. Muller AF, Donnelly MT, Smith CML, Grundman MJ, Holmes GK, Toghill PJ (1996) Neurological complications of celiac disease: a rare but continuing problem. Am J Gastroenterol 91:14301435

12. Alaedini A, Green PHR, Sander G, et al. (2002) Ganglioside reactive antibodies in the neuropathy associated with celiac disease. $\mathrm{J}$ Neuroimmunol 127:145-148

13. Cuoco L, Jorizzo A, Certo M, et al. (1999) Prevalence and early diagnosis of celiac disease in autoimmune thyroid disorders. Ital $\mathrm{J}$ Gastroenterol Hepatol 31:283-287

14. Tursi A, Giorgetti GM, Brandimarte G, Rubino E, Lombardi D, Gasbarrini G (2001) Prevalence and clinical presentation of subclinical/silent celiac disease in adults: an analysis on a 12-year observation. Hepato-gastroenterology 39:462-464

15. Usai P, Usai Satta P, Lai M, Corda MG, Piras E, Calcara C, Boy MF, Morelli A, Balestrieri A, Bassotti G (1997) Autonomic dysfunction and upper digestive functional disorders in untreated adult celiac disease. Eur J Clin Invest 27:1009-1015

16. Gibbons CH, Freeman R (2005) Autonomic neuropathy and celiac disease. Autonomic neuropathy and celiac disease. J Neurol Neurosurg Psychiatry 76:579-581

17. Walker-Smith JA, Guandalini S, Schmitz J, Schmerling DH, Visakorpi JK, on behalf of Working Group of European Society of Paediatric Gastroenterology and Nutrition (1990) Revised criteria for diagnosis of celiac disease. Arch Dis Child 65:909911

18. Feighery C, Weir DG, Whelan A, et al. (1998) Diagnosis of glutensensitive enteropathy: Is exclusively reliance on histology appropriate? Eur J Gastroenterol Hepatol 10:919-925

19. Oberhuber G, Grandisch G, Vogelsang H (1999) The histopathology of celiac disease: time for a standardized report scheme for pathologists. Eur J Gastroenterol Hepatol 11:1185-1194
20. Cornblath DR, Chaudhry V, Carter K, Lee D, Seysedar M, Miernicki M, Joh T (1999) Total Neuropathy Score: validation and reability score. Neurology 53:1660-1664

21. Associazione Italiana di Neuroimmunologia (2004) Diagnostica delle neuropatie periferiche disimmuni. In: Standardizzazione di procedimenti e metodiche laboratoristiche in neuroimmunologia, September, pp 21-26

22. Tursi A, Brandimarte G, Giorgetti GM, et al. (2005) Endoscopic and histological duodenal findings of celiac adult patients on a gluten-free diet: a 2-year follow-up prospective study. Dig Liver Dis 37(Suppl 1):S100-S101

23. Holmes G (1997) Neurological and psychiatric complications in celiac disease. In: Epilepsy and other neurological disorders in celiac disease. John Libbey, London, pp 251-264

24. Luostarinen L, Himanen S-L, Luostarinen M, Collin P, Pirttilä T (2003) Neuromuscolar and sensory disturbances in patients with well treated celiac disease. J Neurol Neurosurg Psychiatry 74:490 494

25. Volta U, De Giorgio R, Petrolini N, et al. (2002) Clinical findings and antineuronal antibodies in celiac disease with neurological disorders. Scand J Gastroenterol 37:1276-1281

26. Briani C, Riggero S, Zara G, et al. (2004) Anti-ganglioside antibodies in children with celiac disease: correlation with gluten-free diet and neurological complications. Aliment Pharmacol Ther 20:231235

27. Mauro A, Orsi L, Mortara P, Costa P, Schiffer D (1991) Cerebellar sindrome in adult celiac disease with vitamin E deficiency. Acta Neurol Scand 84:167-170

28. Giorgetti GM, Tursi A, Iani C, et al. (2004) Assessment of autonomic function in untreated adult celiac disease. World J Gastroenterol 10:2715-2718

29. Ventura A, Magazzù G, Greco L (1999) Duration of exposure to gluten and risk for autoimmune disorders in patients with celiac disease. Gastroenterology 117:297-303

30. Londei M (2001) The external world of gluten and autoimmunity. Gut 49:463-464 